Slavica

bruxellensia

\section{Slavica bruxellensia}

Revue polyphonique de littérature, culture et histoire

slaves

$3 \mid 2009$

Pensée des hommes

\title{
La religion de la paysannerie biélorusse : la métaphysique de l'ordinaire
}

\section{Julja Černjavskaja}

Traducteur : Katia Vandenborre

\section{OpenEdition}

\section{Journals}

Édition électronique

URL : http://journals.openedition.org/slavica/269

DOI : 10.4000/slavica.269

ISSN : 2034-6395

\section{Éditeur}

Université libre de Bruxelles - ULB

Édition imprimée

Pagination : 17-33

ISSN : 2031-7654

\section{Référence électronique}

Julja Černjavskaja, « La religion de la paysannerie biélorusse : la métaphysique de l'ordinaire », Slavica bruxellensia [En ligne], 3 | 2009, mis en ligne le 15 juin 2009, consulté le 19 avril 2019. URL : http:// journals.openedition.org/slavica/269 ; DOI : 10.4000/slavica.269

Ce document a été généré automatiquement le 19 avril 2019.

\section{(c) (i) (9)}

Les contenus de Slavica bruxellensia sont mis à disposition selon les termes de la Licence Creative Commons Attribution - Pas d'Utilisation Commerciale - Pas de Modification 3.0 France. 


\title{
La religion de la paysannerie biélorusse : la métaphysique de l'ordinaire
}

\author{
Julja Černjavskaja \\ Traduction : Katia Vandenborre
}

\section{NOTE DE L'ÉDITEUR}

La langue originelle de cet article est le russe.

\section{La situation historico-confessionnelle en Biélorussie du $X^{\mathrm{e}}$ au $\mathrm{XX}^{\mathrm{e}}$ siècle}

Avantdeprocéder à l'analysedelareligiontraditionnelle du peuple biélorusse, ilconviendraitd'esquisserbrièvementlesorigines historiques de la situation confessionnelle en Biélorussie, laquelle a été marquée par de fréquents troubles. Ce territoire est passé de nombreuses fois de la Pologne à la Russie et vice versa, impliquant de ce fait une « recoloration » confessionnelle de la population.

Ainsi, quand elle faisait partie de la Rus' de Kiev, la population des terres biélorusses se convertit à l'orthodoxie, et la religion officielle devint un marqueur de conscience, du moins pour l'élite de la société: la paysannerie, c'est-à-dire la majorité, était principalement de confession païenne. C'est notamment pendant la période de la Rus' de Kiev que commença à se constituer sur le territoire de la Biélorussie un panthéon de nečistiki (les divinités païennes), qui plus tard accompagnèrent le Biélorusse tout au long de sa vie. Le rapport à celles-ci était ambivalent, allant de l'inimité à la sympathie.

Quand les terres biélorusses furent intégrées au Grand-duché de Lituanie, la population de Biélorussie occidentale commença dès 1251 à se convertir progressivement au catholicisme conformément à l'oukase de Mindaugas. Du reste, il ne convient pas de 
situer le schisme entre les orthodoxes et les catholiques à ce moment-là : ils gardaient un ensemble considérable de croyances païennes ainsi que les normes et les valeurs de vie, de droit et d'éthique qui en découlaient. En général, dans le mode de vie des paysans, la transformation confessionnelle n'était pas tout à fait consciente : les gens continuaient à recourir à l'aide des nečistiki et à expliquer leurs chances et malchances par les actions de ceux-ci.

La période suivante était celle de l'union du Grand-duché de Lituanie et de la Couronne polonaise (1569). La conséquence en fut une conversion massive et - à la différence de la période précédente - consciente de la noblesse du Grand-duché au catholicisme. Le noble catholique recevait des privilèges juridiques qui lui donnaient la possibilité non seulement de bénéficier des honneurs, mais aussi de s'enrichir. En ce qui concerne les serfs, leur conversion au catholicisme avait un caractère forcé et était déterminé par les volontés du noble (le "pan»). Dans les milieux professionnels et artisans des villes, la «catholicisation » était assurée par l'enseignement en polonais (souvent gratuit) dans les collèges et les écoles jésuites. L'union de Brest-Litovsk (1596) qui tenta de réconcilier les confessions en mettant en place un moyen terme, l'uniatisme, se solda par un échec sur les terres biélorusses, contrairement à l'Ukraine.

Suite au traité de Tilsit en 1807, le territoire ethnique de Biélorussie actuel fut intégré à l'empire russe. À partir de cette époque, l'orthodoxie commença à pénétrer la population aussi brutalement qu'elle en avait été extirpée. Les persécutions du catholicisme et dans l'ensemble du "début d'occidentalisation » de la culture biélorusse acquirent un caractère particulièrement aigu après les soulèvements de 1831 et de 1863.

Ainsi, les perturbations confessionnelles sur les terres biélorusses s'exprimaient « par le fer et par le feu » et portèrent préjudice en premier lieu à la paysannerie, la couche la plus nombreuse et la plus opprimée de la population. Malgré que cette couche fût à peine consciente des raisons des tempêtes qui se déchaînaient sur sa terre, les conséquences considérables de celles-ci se firent sentir sur les conceptions religieuses des paysans biélorusses et, dans l'ensemble, sur leur image du monde. Comme l'a montré notre étude des contes du quotidien ${ }^{1}$, mis par écrit au tournant des XIX ${ }^{\mathrm{e}}$ et XX $\mathrm{XX}^{\mathrm{e}}$ siècles (ici nous allons profiter des notes de l'ethnographe et folkloriste Aleksandr Seržputovski [1864-1940]), une des conséquences de l'opposition confessionnelle sur le territoire de la Biélorussie a été la formation d'une "religion paysanne » originale. Celle-ci s'est construite pas tant sur la base des sermons de l'Église que sur la fusion symbiotique du Biélorusse avec sa terre natale. Ce type local et indigène d'identité (ainsi que le type de religiosité qui lui correspond) est apparu comme le résultat de l'impossibilité de s'identifier à une religion stable $^{2}$ ou à un tout étatique stable.

\section{Bases théoriques et méthodologiques de l'analyse}

Nos recherches sont interdisciplinaires: elles comprennent des éléments d'analyse sémiotico-culturelle, de sociologie du quotidien, mais aussi de folklore et d'anthropologie culturelle. Cela conditionne l'approche générale, qui, par analogie à l'aphorisme du « retour à la nature » de Jean-Jacques Rousseau, peut être définie comme « un retour au texte ». Le texte nous apparaît comme un champ particulier de «compréhension» ou comme un prétexte à l'empathie culturologique.

Ici se pose le problème du rapport entre la « compréhension » et « l'interprétation », et ce notamment dans la question de ses limites (question posée par Susan Sontag dans Contre 
l'interprétation en 1966). C'est pourquoi, en matière d'outil méthodologique de base, nous sommes guidée par le principe "d'idéal-type » de Max Weber ${ }^{3}$. Celui-ci considère que l'analyse la plus méticuleuse n'est pas capable de saisir tous les aspects du phénomène étudié. Par conséquent, la prétention à l'authenticité et à l'universalité ne doit pas être le but de l'analyse, mais bien la création d'un type idéal de «fantaisie scientifiquement disciplinée » (termes de Weber). Toutefois, cette fantaisie ne doit pas devenir « lâche » ou "étouffante " (termes de Sontag) : elle est obligée de proposer un certain "germe " invariable. De cette manière, l'idéal-type est le résultat d'une analyse scrupuleuse des situations types, des rapports sociaux ainsi que des modèles de comportement et de perception des agents de l'action et de l'interaction, ce qui nous apparaît comme fondamental. Nous considérons que de tels modèles se reflètent dans les textes du folklore, qui, à leur tour, se présentent comme des segments d'un texte plus large, un texte du quotidien des paysans biélorusses.

Nous adopterons une approche double qui est celle, d'une part, de l'anthropologie culturelle et, d'autre part, du folklore. Du point de vue de l'anthropologie culturelle, nous partons du principe classique "at a distance $»^{4}$ de l'école "Culture et personnalité », qui propose l'étude de la culture à distance. Mais si Ruth Benedict, Geoffrey Gorer, Margaret $\operatorname{Mead}^{5}$ et de nombreux autres chercheurs ont analysé la culture de pays qu'ils ne fréquentaient pas par le biais des textes issus de cette culture, alors dans notre cas la distance entre le chercheur et l'objet est encore plus insurmontable puisqu'elle s'avère temporelle (les contes dont nous parlons ont été écrits entre les XVII ${ }^{e}$ et XIX ${ }^{e}$ siècles et mis par écrits par des ethnographes à la fin du XIXe et au début du XXe siècle).

Puisque nous nous basons sur des sources folkloriques, il s'est avéré nécessaire de se référer aux travaux de mythologues et de folkloristes. Les travaux de Vladimir Propp, d'Eleazar Meletinskij, de Sergej Nekljudov, fondateur de l'école moderne du " postfolkore », dont l'activité se développe au sein de l'Université de sciences humaines d'État russe de Moscou (RGGU) ${ }^{6}$. Le recours à la méthodologie de ces auteurs se justifie par le fait que, travaillant dans la tradition folklorique, ils placent leurs recherches dans un contexte anthropologique bien plus large que l'étude traditionnelle du folklore. Ils étudient le folklore comme un réservoir d'habitus qui déterminent le tableau du monde, les modèles quotidiens de vie ainsi que les stratégies de comportement et d'action des représentants de la culture.

La théorie de l'habitus de Pierre Bourdieu est pour nous fondamentale. L'habitus est un système de dispositions transposables (d'orientations, de valeurs, de schémas de perception et d'action), qui se structurent de l'extérieur (par le pouvoir, la situation socioculturelle, la langue, etc.) et qui en même temps structurent "de l'intérieur ${ }^{7}$ le champ de la culture. Le concept d'habitus est irremplaçable pour l'analyse de textes de la culture traditionnelle : il indique le mécanisme de conservation de cette tradition ainsi que des changements qui y ont été apportés par les gens. «L'habitus du sacré » est, dans n'importe quelle culture, un des plus importants. L'agent de la culture le traite à la fois comme le plus haut dessein et comme une activité quotidienne. Le but de l'article sera de définir l'habitus du sacré dans la culture traditionnelle biélorusse.

Pour ce faire, notre étude s'appuiera sur des textes folkloriques et plus particulièrement des contes biélorusses du quotidien. Notre choix a été motivé par une série de raisons. Premièrement, le folklore donne la représentation d'un ordre stable des choses et, par conséquent, du quotidien, compris comme une sphère spécifique du rapport entre le sacré et le séculier. Deuxièmement, c'est précisément dans le folklore (et surtout dans les 
contes) que se transmet concrètement le lien entre les rôles social et linguistique, mais aussi sacré et profane, des agents. Troisièmement, les contes révèlent des modèles typiques de comportement qui font office de point de repère pour les nouvelles générations. Enfin, le plus important: les contes donnent une image vivante du héros. Dans notre cas, il s'agit de l'image de Dieu, des saints, des prêtes et des « saints hommes ». Les sources servant de matériel pour l'article sont constituées des contes réunis par Seržputovskij ainsi que d'une série de textes de la même époque provenant des recueils d'autres chercheurs.

\section{L'image de Dieu dans le conte biélorusse : mystique et pragmatique}

La religion traditionnelle du Biélorusse se construit autour de son lien avec la terre, comprise comme un capital tant économique que symbolique. C'est de là que proviennent non seulement les représentations païennes ${ }^{8}$ liées aux esprits impurs (les nečistki que sont les gumennik ${ }^{9}$, ovinnik ${ }^{10}$, hlevnik $k^{11}$, rusalki ${ }^{12}$, vodjaniki ${ }^{13}$, etc.), mais surtout l'image du Dieu « terrestre », " paysan ».

Dans la conscience populaire, l'image de Dieu est polysémique. Les contes contiennent une image « déiste » de Dieu, qui a créé la terre ainsi que tout ce qui s'y trouve pour les hommes; pour lui-même il a créé le ciel, où il s'est caché des péchés humains. Il existe aussi une image « apocryphe » de Dieu, propre à de nombreuses cultures traditionnelles : «Dieu est juste, mais très fâché. Il ne pardonnera jamais le mal. ${ }^{14}$ Ici le problème de théodicée est résolu par des « moyens locaux » : « Si Dieu le voulait, il anéantirait tous les diables, tous les esprits malins et l'abomination, il ne le fait cependant pas pour que les gens aient peur, sinon ils oublieraient Dieu lui-même. $\aleph^{15} \mathrm{Il}$ y a également une image «transcendante » de Dieu : la légende insiste sur le fait qu'il y a simplement des dieux et un Dieu au dessus des dieux ${ }^{16}$. À la différence des "simples dieux ", le Dieu qui est audessus des dieux ne possède pas d'apparence anthropomorphe, il est plutôt identique au Destin, au Fatum.

Toutefois, l'image du «Dieu terrestre, paysan» est la plus répandue. La création du monde et de l'homme, l'accoutumance de l'homme au travail et l'établissement d'un ethos du travail, constituant le cœur de la vie du paysan biélorusse, sont notamment liés à celle-ci. «Jadis les gens étaient bêtes; ils ne pouvaient rien faire ${ }^{17}$, c'est pourquoi le Christ et la Mère de Dieu se sont rendus sur terre et ont enseigné « comment vivre sur terre, comment cultiver la terre pour que chaque chose profite à l'homme $»^{18}$. Le travail tout comme l'esprit pratique sont jugés agréables à Dieu.

L'exemple de Dieu se ressent dans la concrétisation de l'activité humaine. Pendant l'âge d'or, Dieu (le Christ) est venu chez les hommes et, « comme un frère, il leur enseigna comment vivre sur terre et comment engendrer des enfants, engendrer et élever des enfants et combler la terre... Les gens, comme des fleurs, fleurissaient, vivaient comme des oiseaux dans le ciel ou des poissons dans l'eau. Ils se servaient de ce qu'ils trouvaient et ne craignaient ni le malheur, ni la pauvreté. Mais qu'est-ce que la vie? Ainsi vivent les bêtes des forêts et les morceaux de bois. L'ayant remarqué, Dieu se mit à apprendre aux hommes comment attraper plus facilement du gibier, comment se faire des vêtements, comment s'abriter lors des intempéries, comment attraper les poissons, des bêtes, des oiseaux. Et les hommes comprirent que celui que Dieu avait pourvu de raison pouvait 
faire ce qu'il voulait $"^{19}$.C'est cependant le travail qui est reconnu comme le plus honorable sur terre : il est réformateur comme les actions de Dieu qui a créé la terre. De cette manière, Dieu sanctionne « le rapport au monde tout comme à l'exploitation de la terre $\rrbracket^{20}$ et, par conséquent, un respect particulier pour le travail des paysans.

Dans le conte "Le chasseur et le pêcheur ", deux frères refusaient de suivre le même parcours que leur père : l'agriculture. L'un devint chasseur : « Les gens allaient au travail alors que lui, il épaulait son fusil et, hop, allait dans la forêt ou le marais. Il traînait là toute la journée et revenait à la maison les mains vides, affamé comme un loup. $»^{21}$ Ledeuxième frère«restaitassisjouretnuitauborddelarivièreetpêchait des poissons. Il attrapait un tas de tout petits poissons : de quelle utilité étaient-ils pour le ménage ? $»^{22}$ Iln'yeutriend'étonnant à ceque,quandlepèresefitvieux, lapropriété devintsidélabréequ'iln'avaitplus rien à offrirau sage voyageur qui se présenta. Le pèlerin partagea sa propre aumône avec les fils et dit avec du bon sens : « Ainsi, vous voyez, il [le père] est vieux, il s'est nourri et vous a nourri grâce à son exploitation; sans cette exploitation, vous, jeunes et robustes que vous êtes, ne pouvez pas survivre. $»^{23}$ Remarquonsquesouslestraitsde«sage », de «pèlerin » se cache souvent Dieu en personne.

Ainsi, letravail peutporter sesfruits; s'il ne lepeut, il n'estpasconsidéré commevéritablecar, étant donné parDieu, il dépend, bien que dansunemoindremesurequel'agriculture, deseffortsjournaliersetdelaconséquencedesactions. C'est pourquoi tant le chasseur que le pêcheur (et d'autres professions de village comme le forgeron, le meunier, etc.) sont constamment associés au diable dans la conscience populaire, car ils font contrepoids au paysan (ou comme il s'appelle lui-même le «moujik»), qui accomplit un travail saint. Il est probable que le rejet de l'image de « l'étranger » (du pan, du citadin, du soldat, du juif, etc.), liée à des professions non-champêtres, joue ici un rôle significatif.

En raison de l'égoïsme humain, Dieu apparaît de plus en plus rarement sur terre sous sa " vraie » apparence traditionnelle et il aide de plus en plus rarement l'homme dans ses détresses : « Avant, quand les hommes respectaient Dieu et lui obéissaient, Il leur donnait tout ce qu'il fallait, mais maintenant les jeunes ont complètement oublié Dieu, et c'est de là que viennent tous les malheurs... ${{ }^{24}}^{24}$ Par contre, si l'homme croit en Dieu et suit ses conseils, il reçoit une récompense. Comme nous l'avons déjà noté, au moment où ces

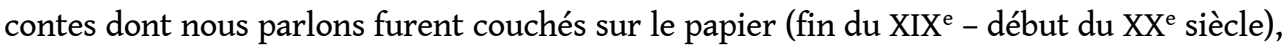
Dieu est représenté, en règle générale, sous la forme d'un misérable pèlerin, d'un vieillard. Il voyage parce qu'il est nécessaire à chacun et qu'il doit partager son attention entre tous. C'est pourquoi il existe une représentation selon laquelle les rapports entre Dieu et les hommes se développent non pas avec l'aide de l'Église, mais «face à face». C'est de là que vient le sujet le plus populaire des contes, qui est justement lié au contact direct entre Dieu et l'homme.

Dans un conte, une femme qui avait donné du kvas à Dieu (qui s'était présenté sous la forme d'un pèlerin) est guérie de sa stérilité. Elle voit en rêve une haute montagne (image du paradis). Trois jeunes filles s'y promènent : ce sont trois déesses du Sort (Doli). L'une tresse une couronne de chardon et de sonchus, la deuxième d'épines et de branches, la troisième de seigle et de blé avec des fleurs. Les jeunes filles débattent pour savoir à laquelle d'entre elles échoira l'enfant conçu. Finalement, elles décident de faire comme Dieu l'ordonnera. Dans ce but, elles jettent une fleur en l'air. La troisième l'attrape. Un petit garçon naît et connaît une vie heureuse de paysan ${ }^{25}$. Ici s'entrelacent étroitement des symboles culturels populaires: la récompense pour la bienveillance envers un faible 
vieillard (en effet, la femme ignore qu'elle donne du kvas à Dieu en personne), envers Dieu et le Sort, le fatalisme de ce qui est écrit « dans la destinée », l'appel à Dieu dans une situation de choix. Deux moments méritent une attention particulière.

Le premier tient au fait que Dieu est plus fort que le Sort. Il est comparable aux mythes grecs où le Destin antique est au-dessus des dieux. Il est probable que les rapports inversés de Dieu et du Sort soient liés au monothéisme chrétien. L'immense force de Dieu peut s'expliquer par le fait que le Sort se présente sous la forme d'une femme et Dieu sous l'apparence d'un homme rendu sage par la vie. Le deuxième moment concerne les modèles de la vie future, symbolisés par les couronnes des jeunes filles : la couronne de sonchus et de chardon présume une vie de pêcheur ou de voyageur ; la couronne d'épines et de branches évoque celle de chasseur ; la couronne de seigle, de blé et de fleurs celle de paysan. Dieu fait comprendre que c'est précisément au dernier type d'activité que va sa préférence. Remarquons que dans la couronne sont entrelacées des fleurs : cela signifie que le travail du paysan ne suppose pas seulement des efforts, mais aussi la joie de vivre dans un monde merveilleux :

Comme nous le voyons, Dieu ne donne pas ses recommandations de manière littérale. Il préfère un "chemin détourné ", une forme symbolique d'expression. En vérité, c'est en cela que se trouve le mysticisme, que se fait sentir l'essence ésotérique, laquelle se manifeste à travers les normes pragmatiques et prosaïques de la vie champêtre.

Lefaitsuivantestluiaussiremarquable :

malgréle«déplacement »

purementpaïendeDieusurterre,

cescaractéristiquesdel'imagedeDieusereflètentdanslaconceptiondumondedelapaysanneriebiélorusse.

Celles-ci sont propres au christianisme canonique: Dieu est sage, tout-puissant, omniscient et surtout bienveillant. L'homme a été créé à Son image. Dieu n'a pas seulement créé l'homme, Il a aussi créé le monde: la création biblique du monde se concrétise à peine, émanant d'un mode de vie unique, qui est un signe à la paysannerie biélorusse mais ne s'oppose pas à l'enseignement du Christ.

\section{Les médiateurs « entre le monde et la vérité » 27}

À l'analyse du phénomène de dévotion populaire du Biélorusse, le rapport ironique et souvent négatif aux serviteurs du culte et même aux saints canoniques saute aux yeux. Il est probable que cela soit lié à leur rôle d'intermédiaire :

Il est remarquable que les prêtres orthodoxes et catholiques soient mentionnés ensemble. La raison de la méfiance envers eux est évidente : comme nous l'avons montré plus haut, pendant plusieurs siècles, le paysan biélorusse a plus d'une fois été contraint de se convertir soit à l'une soit à l'autre religion. Le modèle de l'office divin, sa langue et surtout l'esprit même du culte changeaient. C'est pourquoi la relation « face à face » est devenue le seul lien possible avec Dieu : en venant à l'homme « comme un frère », Dieu en personne lui donnait cette possibilité.

La caractéristique la plus fondamentale du prêtre est paradoxale, car on dit qu'il fréquente les esprits maléfiques :

En réalité, la cause principale d'une telle antipathie est très ancienne, elle est liée à la partition mythique du réel et du surnaturel, à la bénédiction des mondes où le serviteur du culte joue le rôle de médiateur: dans les contes, le prêtre apparaît plus que tout comme un maillon reliant non seulement les mondes d'en bas et d'en haut, mais aussi le 
troisième, le «nav' « ${ }^{30}$. Cela explique le sujet de nombreux contes, dans lesquels il est question du séjour constant $\mathrm{du}$ (ou des) diable(s) à l'église. En fait, la spécificité " livresque » du travail de "cet homme instruit dans le village $»^{31}$ fait que l'on lui attribue un lien avec les forces maléfiques: dans la culture traditionnelle, la connaissance, et tout particulièrement la connaissance sacrée, recèle un certain danger, constitue une forme de transgression. C'est pourquoi, d'après les représentations paysannes, il ne sert à rien au saint homme d'aller à l'église.

En effet, le prêtre, de la même manière que les autres créatures liées aux forces de l'audelà, éveille la peur. Le rire est un moyen de freiner cette peur. Ainsi, l'image du serviteur du culte dans les contes est comique voire satirique. Le bon sens du paysan ne peut ignorer l'inadéquation de l'image réelle du pope (assez souvent rusé, âpre au gain et corrompu) et de son rôle d'intermédiaire entre les mondes. De plus, le sentiment de dignité du moujik, qui comprend littéralement sa place «en-dessous de Dieu», ne lui permet pas de prendre au sérieux ces intermédiaires. À cela s'ajoutent d'autres éléments qui jouent un rôle non négligeable: la proximité du pope avec les forts de ce monde, principalement avec le pan, la figure la plus négative du folklore biélorusse ; le fait que le pope et le prêtre reçoivent de l'argent réel, des biens; la possibilité qu'il a d'avoir une relation avec la femme d'un autre en «cadeau pour sa sainteté », etc. De ce fait, une telle tournure est caractéristique des contes :

À cet égard, le pope ne se différencie en rien du prêtre.

$\mathrm{Au}$ vu d'un tel rapport à la prêtrise, l'Église ne joue pas un rôle très important dans la vie du paysan. Cela explique la description ironique voire satirique du service religieux dans les contes :

Lefaitd'aller à l'égliseestunattributsouhaitablemaispasobligatoiredelaviedupaysan, ilabeaucoupmoinsdesignificationqueletravail : «Le travailleur n'a pas le temps d'aller à l'église, en plus les travailleurs des popes n'y vont jamais. $\|^{34}$ Leschercheursetlesmémorialistes (Mikalajulaščyk, VladimirKorotkevič, AleksandrBogdanovič, etc.) en témoignent: sienhiver, pendantlapériodecreuse, lesgensallaient à l'église, en été, ilsn'avaientpasletemps. Parcontre, il faut remarquer quelacommunauté russeallait à l'églisemême pendant la période la plus chaude de la moisson.

Les images de saints dans les contes ne sont pas univoques. D'un côté, la mission du saint est de demander à Dieu la clémence pour les gens (saint Nikolaj et la Mère de Dieu remplissent très souvent cette fonction). D'un autre côté, les images de saints sont souvent trop terrestres. Saint Pëtr, par exemple, aime se remplir le ventre de nourriture gratuite, il vole même du pain à saint Boris ou, dans une autre variante de la légende, au Christ («D'où sont venus les champignons »). En général, le vol par les saints est un thème fréquent dans le folklore. Les saints n'ont pas seulement des défauts (fainéantise, égoïsme, etc.), ils accomplissent en plus des choses qui sont considérées par le peuple comme de véritables péchés. Dans le conte « Le mal passe, et ne dépasse pas le bien », les saints refusent de rendre visite à une pauvre veuve qui compte sur eux pour redonner vie à son fils qui se meurt. Ils préfèrent rester couchés sur le poêle dans une maison riche, « seul le Christ eut pitié et s'en alla dans la boue jusqu'au bout du village, chez la pauvre veuve. $\rrbracket^{35}$

L'essence terrestre des saints se révèle dans le fait qu'ils sont souvent égaux aux hommes voire plus faibles qu'eux. Il existe une série de contes où l'on punit les saints, notamment 
en leur donnant les verges. Pour le paysan, le saint est un moujik pris, grâce à son succès, sous la protection de Dieu. À la différence du moujik, le saint peut accomplir des miracles, mais secondaires : ils ne résultent pas de la nature particulière du saint, mais du fait que Dieu les leur a appris. Souvent, les miracles ne se produisent pas sur la volonté du saint, mais, au contraire, de son non-vouloir, dans le but de l'exhorter. Ainsi, d'après le conte "D'où sont venus les champignons ", les champignons sont apparus grâce à l'égoïsme et la faiblesse d'esprit de saint Pëtr qui n'avait pu s'empêcher de chiper du pain :

Soulignons d'emblée que le Christ apparaît toujours en qualité de Dieu paysan, de maitre diligent: ses actions «servent» les gens. Observons les contextes dans lesquels apparaissent les motifs chrétiens. Le Christ ne punit pas ni même ne conscientise Pëtr. Étant donné que la raison d'une telle bonté n'est en rien explicitée dans le texte, la possibilité de l'expliquer est placée de manière latente dans la culture et se présente comme un "savoir original de fond »: la maxime du pardon chrétien est entrée dans la pratique spirituelle du peuple. La manière de faire le bien est remarquable: elle n'implique pas de « révolution » radicale et vient de l'idée que tout mal peut être changé en bien au moyen de pensées bonnes. Cette transformation du mal en bien (le pain volé se métamorphose, par l'enchantement du Christ, en nourriture pour tous) renvoie sans aucun doute aux miracles du Christ. Toutefois, le plus important ici tient sans doute au choix que fait le Biélorusse dans sa façon de lutter contre l'injustice. En effet, que ce soit dans les contes ou dans la vie ordinaire, il opte précisément pour des pensées bonnes et, dans l'ensemble, pour un modèle détourné de comportement. Ceci est possible car le mal s'autodétruit par la force seule de sa nature diabolique. «Il n'y a rien à faire, il faut subir car tu n'arriveras pas à vaincre le mal. $»^{37}$ Nousprésumons quec'estdelà quevientlagrandepatience, historiquementcélèbre, duBiélorusse. Malgré la relativement faible religiosité du Biélorusse, on ne peut le qualifier d'athée ou d'agnostique. Il croit, mais cette foi est spécifique.

\section{Deux formes de foi}

En reprenant le titre d'un des travaux de Martin Buber ${ }^{38}$, nous pouvons dire que deux formes de foi ont coexisté (et, dans une certaine mesure, existent encore aujourd'hui) sur le territoire biélorusse. La première est une manière de se comporter au quotidien qui consiste essentiellement à suivre les normes chrétiennes au quotidien. Le deuxième type de foi est personnifié par la figure du "saint homme». Les deux formes de foi se réunissent dans une conception globale selon laquelle le monde est multiple, que son remplissage est symbolique voire mystique et que la principale fonction de l'homme est de déchiffrer les signes divins dans la vie de tous les jours. La distinction des deux formes de foi et des modèles de conduite leur correspondant peut se comprendre comme suit : la première (celle d'une morale ordinaire) se base sur l'acceptation du mal comme une part nécessaire de la vie et postule la patience comme réaction au mal, la deuxième se construit sur la distinction intuitive et claire du bien et du mal.

Lepremiertypedefoireposesurl'idéefatalistedel'invincibilité

dumalparl'hommeet,enpartie,surlaconvictionque le mal est condamné à se détruire. Dans les contes, c'est exprimé de manière univoque depuis les simples histoires d'homme cupide et lâche (ou de diable) qui se porte préjudice à lui-même sans le vouloir jusqu'à celles qui mettent en scène des massacres de masse entre les membres d'une famille de bandits ( « La tribu des loups »). Dans la vision du paysan biélorusse, il est vain de punir le 
mal du dehors : premièrement, il s'autodétruit dans un délai fixé non pas par l'homme mais par Dieu; deuxièmement, la tentative de l'extirper par la force produit un mal encore plus grand : «Il est bien connu que si tu détruis le mauvais, le bien disparaît. »"

Ainsi, poussé par les meilleurs motifs, le héros du conte "Les massepains " décide d'obtenir l'égalité pour tous; cependant, au final de ses actions, les gens se retrouvent privés de plus de droits encore. L'idée centrale de ce conte, comme de tant d'autres, est la suivante: les tentatives de transformations sociales radicales par le biais d'actions directes mènent à la violence (ce n'est pas un hasard si l'auxiliaire du héros dans ce conte n'est pas Dieu, mais le diable). En fait, le mal n'a pas de localisation claire, il est dispersé dans le monde, mêlé au bien et, de ce fait, il est impossible de le distinguer : « Les gens ont raison lorsqu'ils disent qu'on ne devinera jamais où est le mal et où est le bien... » ${ }^{40}$

La deuxième forme de foi est éloignée du quotidien. Elle est représentée par des images de gens proches de la sainteté (dans les variantes de contes «L'homme saint », « Le groin de cochon », « Le pope et l'ermite », etc.).

Le héros du conte "L'homme saint " se trouve dans une situation marginale, entre les deux formes de foi. C'est un homme calme vivant à l'extrémité du village, ne refusant à personne ni nourriture ni logement. Les voisins le considèrent comme un saint parce qu'il est bon et qu'il va régulièrement à l'église. Un jour, un vieillard qui allait de par le monde passa chez lui. Le «saint homme" se rendit avec lui à l'office. Pour cela, ils devaient traverser la rivière à gué. Le vieillard (un saint homme ou Dieu en personne, mais, dans le conte, ce n'est pas évident à distinguer) frappa l'eau avec un bâton et elle s'écarta. Prenant conscience de la noble origine de l'hôte, le moujik refusa d'entrer dans l'église : " Je ne mérite pas d'aller avec toi dans l'église de Dieu. Vas-y seul, je resterai à la porte. $»^{41}$ Pour son humilité, le vieillard le gratifia du don de marcher sur l'eau sans se mouiller et sans être vu. Cependant, les gens ne remarquèrent pas ces changements puisqu'ils ne voyaient pas le héros quand il se trouvait dans les alentours de l'église. Dans le village le bruit courait que le saint homme avait cessé d'aller à l'office. Il n'était vu que par les diables qui vivaient dans l'église. Leur fonction était d'inscrire les péchés humains sur une peau de bœuf afin de les révéler aux forces d'En-Haut. En voyant leur zèle, notre héros sourit, il devint visible et corporel à tel point qu'en revenant de l'église, il s'enfonça dans la rivière jusqu'à la ceinture. Toutefois, seul lui remarqua sa chute morale : c'est précisément après cela que les gens recommencèrent à le considérer comme saint (car ils le virent de nouveau à l'église). Le message du conte est le suivant : « Il est bien connu que les gens ne considèrent un homme comme saint que quand il pèche car le cœur de l'homme est fait de ténèbres, personne ne voit sa conscience, excepté Dieu. ${ }^{42}$ Mais la pensée centrale du conte est plus profonde. Que reçoit l'homme en récompense pour sa « sainteté »? Un don non applicable dans la vie. Il n'est récompensé « par rien » puisque la récompense matérielle lui pèserait dans la vie de tous les jours. Remarquons que notre héros demeure, dès le début, à l'écart, loin du voisinage. Rappelons qu'il est la personnification de l'imperceptible. Il souligne symboliquement son invisibilité : il devient une « âme » dans la vie. Le saint ne peut résider dans le monde. Il est plus facile à l'invisible de se maintenir à la hauteur de l'ascèse. Le rapprochement vers les hommes et les institutions humaines (même cultuelles) est lourd de péché. Il leur apparaît comme un rire déplacé. 


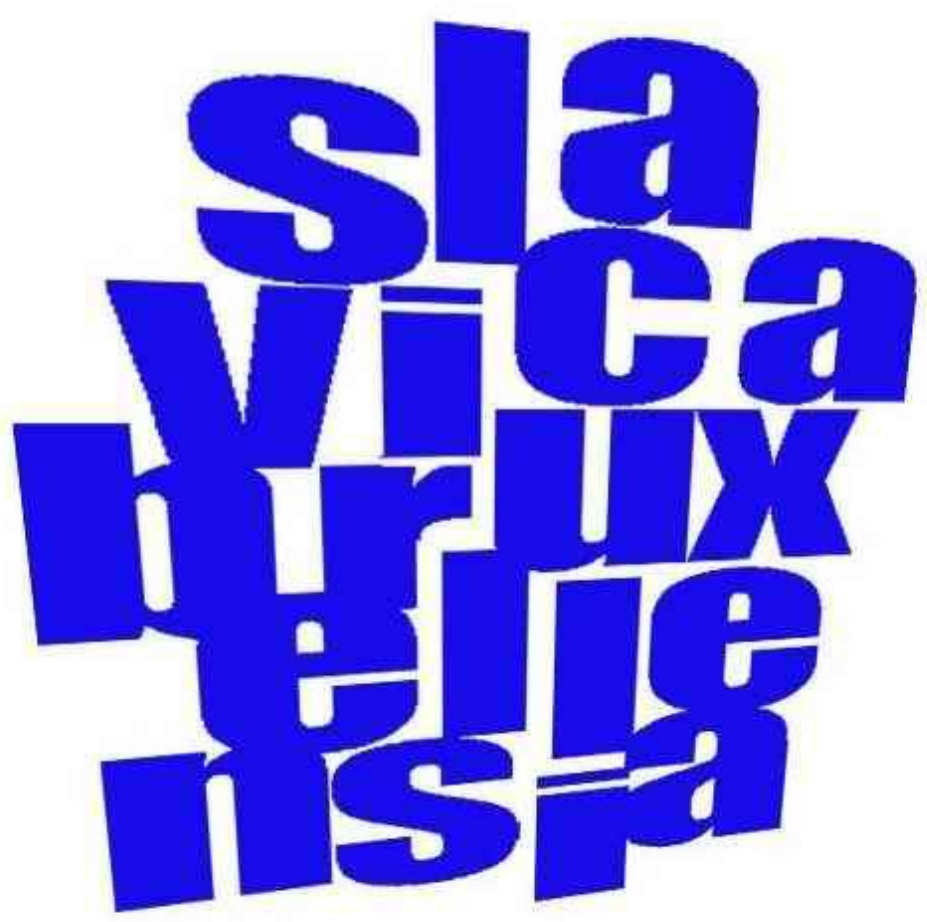

Dans une

autre variante du conte, la situation est exprimée de manière plus précise : le héros ne rencontre pas de vieillard, n'a jamais fréquenté l'église et prie de façon païenne à la maison. Cela met en évidence le fait qu'il est saint précisément jusqu'au moment où il va à l'église. La preuve de sa sainteté est la même que dans la première variante : la capacité de traverser la boue sans se salir. Cela vaut la peine qu'il aille à l'église, voie le diable et sourie quand il se salit dans la boue. Toutefois, le final du conte donne de l'espoir : le pope lui permet de prier à la maison car là-bas sa prière est plus agréable à Dieu. Remarquons que cet homme ne possède pas les merveilleuses qualités du héros du premier conte. La seule preuve de sa sainteté réside dans le fait qu'il ne se salit pas. Par contre, son étrangeté est accentuée dans sa caractérisation « d'imbécile heureux » ${ }^{43}$.

L'étrangeté du héros est encore plus soulignée dans le conte «Le pope et l'ermite ${ }^{44}$. Depuis son enfance, il se distinguait de son entourage : dans sa jeunesse, il n'était pas étranger aux tendances révolutionnaires, puis il devint guérisseur. Il avait une chemise qui « ne portait pas la honte »; il priait, en sautant par-dessus le puits et en répétant : « À toi, Dieu, à moi, Dieu ». Il était capable d'aller sur l'eau sans se mouiller les pieds, à la différence du pope qui était venu chez lui en le sermonnant et qui avait failli se noyer dans une flaque. De plus, il soignait les gens et les instruisait, ce qui le distinguait aussi des autres.

En somme, les contes nous proposent trois images de la sainteté. Le premier héros est bon, hospitalier, pieux et respectueux de la vieillesse. Un tel modèle de conduite idéale est, en principe, accessible à tous. Par contre, cet homme ne supporte pas l'épreuve : la récompense ne lui profite pas. Le deuxième ne possède pas les mérites du premier. Le seul qu'il partage est la prière particulière. Malgré le fait qu'il commette le même péché que le héros précédent, il garde l'espoir de retourner à la prière "domestique ». On suppose qu'après cela le don miraculeux lui reviendra. Quant à l'ermite, sa conduite n'entre pas dans le champ des valeurs du peuple visiblement parce qu'il ne va pas à l'église. Ici, deux moments sont particulièrement remarquables : premièrement, tous les trois sont réunis 
par la capacité de marcher sur l'eau; deuxièmement, l'homme le plus estimable apparaitt comme le moins saint. La racine de sa tragédie est sans doute l'absence d'indépendance : il est doté de qualités merveilleuses, mais, en principe, ne se distingue pas de ses voisins. Ce n'est pas un hasard que ce soit précisément lui qui soit récompensé par un don miraculeux, tandis que les deux autres en jouissent depuis le début. Ils se sont choisi une prière dans laquelle ils peuvent réaliser leur originalité, sans ressentir la dépendance de la société. Autrement dit, il n'est pas simple pour le saint homme d'être bon : il doit avoir le courage de vivre selon ses propres mesures.

Le saint est un homme capable de distinguer le bien du mal. Dans la première variante, il est doué de cette capacité en récompense pour son comportement digne; dans la deuxième, il la possède dès le début. Il est significatif que dans les deux versions de conte, il est le seul qui voit le ou les diable(s) dans l'église. Outre cela, il comprend la nécessité de sa (ou leur) présence précisément dans ce lieu : la raison en est la complémentarité du bien et du mal. C'est pourquoi même le saint homme n'est pas en droit d'extirper directement le mal.

Le seul moyen que le conte préconise pour combattre le mal est de lutter avec soi-même. Penchons-nous encore sur une image de l'homme idéal, telle qu'elle est présentée dans le conte «Le groin de cochon ». Le héros principal est le fils d'un apiculteur ${ }^{45}$, lequel était perçu comme différent. L'apiculteur vivait retiré, «seul comme un poteau. Les voisins disaient de lui qu'il n'était pas normal parce qu'il il ne vivait pas comme les gens. $»^{46} \mathrm{La}$ mère du héros se distinguait par son goût pour la solitude, son amour pour la nature et son inadaptation au quotidien: "On la demandait en mariage et elle ne voulait pas en entendre parler, répliquant qu'elle n'aimait que les fleurs et les abeilles de Dieu. $\aleph^{47} \mathrm{Le}$ fils des deux ermites qui s'aimaient était un beau jeune homme. En raison de sa beauté, les deux tsarines (Ekaterina II et la «tsarine polonaise ») se battaient pour le jeune homme. Le jeune homme supplia Dieu de lui prendre sa beauté et d'arrêter la guerre: en vain. Ekaterina, victorieuse, ne rencontra cependant pas le bonheur : le visage du jeune homme se changea en groin de cochon au moment où l'impératrice voulut l'embrasser. Elle eut tellement peur qu'elle en mourut. Et le jeune homme, qui garda cette apparence de cochon, s'installa dans la forêt, instruisit et soigna les gens. « Et ils se mirent tous à dire : "Malgré son groin, il est un saint homme." $»^{48}$ Le saint homme peut donc se sacrifier, en anéantissant le mal (qu'est ici la beauté) non pas du dehors, mais seulement à l'intérieur de lui-même.

Il est significatif que tous les « saints hommes » vivent loin de leurs voisins, qu'ils ne sont pas semblables aux autres et capables de faire des miracles. Ce n'est pas un hasard si la vie du saint au groin de cochon soit proche du canon des vies de saints. Les contes laissent transparaître l'idée que seul un homme qui se distingue des autres peut améliorer le monde soit en instruisant, soit en soignant, soit de manière plus « visible » (rappelons la mort de Catherine).

De cette manière, dans le conte biélorusse, la différence entre les deux formes de foi s'inscrit très clairement : la justice du quotidien et la connaissance ésotérique qui se base sur la révélation. Du reste, il n'est pas tout à fait légitime de limiter la version populaire biélorusse de la religion exclusivement à ces deux variantes. Il existe un troisième aspect de la foi populaire qui réunit ces deux formes extrêmes: la recherche du contenu métaphysique dans la vie de tous les jours. 


\section{La métaphysique et le quotidien}

Dans les contes biélorusses du quotidien, il est remarquable que les héros expriment rarement des jugements catégoriques. Aussi, dans une situation de conflit, le paysan gagne-t-il le combat grâce au fait qu'il n'agit jamais ouvertement. Des moyens divers sont alors mis en œuvre: la ruse, la dissimulation, le louvoiement, etc. Dans «Les massepains ", nous avons montré que ce modèle concerne non seulement des situations particulières, mais aussi le rapport aux changements radicaux en général. Le chemin détourné est privilégié au détriment des actions directes, même quand elles sont entreprises au nom du bien. Un tel modèle de conception du monde et de comportement s'est sans doute formé en conséquence de la vie privée de tout droit qu'était celle des paysans sous le servage. C'est de là que vient le « mysticisme paysan », original en soi, qui suppose que chaque phénomène a un double-fond : pragmatique et sacré. Il en découle un style de pensée «à la Ésope » qui crée une métaphysique particulière, parfois bien plus raffinée, qui détruit le stéréotype du Biélorusse pragmatique, peu enclin aux matières supérieures ${ }^{49}$.

Comme nous l'avons montré plus haut, le paysan considère que le chemin détourné du raisonnement et de l'action a été donné par Dieu. La tâche de Dieu est de «coder » le monde, celle de l'homme est "d'enlever les scellés » du sens empaqueté dans une forme symbolique et de démasquer l'impératif de l'action et du comportement. Dans cette tâche de décodage, de tous les personnages du conte biélorusse du quotidien, ce ne sont pas les hommes instruits qui réussissent, mais l'unique disciple de Dieu : le moujik.

Dans le conte «Le moujik et le fils du pan», un paysan entre en conflit avec un étudiant en voulant déterminer lequel des deux avait le plus grand savoir. Tandis que l'étudiant expliquait le tonnerre et l'éclair par l'électricité, le moujik les voyait comme la puissance de Dieu. Pour le jeune «savant ", le feu dans le poêle restait un phénomène naturel, pour le moujik c'était la beauté même; le chat qui se lavait avec sa patte ce n'était pas animal, mais la pureté même, la soupente la hauteur, l'eau la grâce. À la différence du jeune homme, nourri au lait des Lumières et vivant dans le monde limité des lois de la nature, le moujik voyait le double-fond de chaque phénomène.

Dans ce contexte, le fait le plus curieux est que le pragmatisme du Biélorusse des contes constitue une image paradoxale sur la base de la métaphysique. Le travail de la terre est sanctionné par Dieu, le laboureur, et c'est pourquoi la moisson n'est pas seulement perçue comme la garantie d'une relative satiété mais aussi comme un miracle. Le paradis n'est pas perçu comme un jardin, par contre le jardin cultivé par le paysan est comme le paradis ${ }^{50}$. C'est pourquoi, dans la conscience populaire, la nécessité du travail acquiert un caractère bien plus sacré que le fait d'aller régulièrement à l'église. Et finalement, Dieu en personne est pragmatique : ses intentions, qui à première vue peuvent paraître absurdes, possèdent toujours un plan solide et précis et, quand son plan est violé, Dieu ne dédaigne pas refaire la création ratée. Ainsi, par exemple, le diable, s'étant assis sur la charrue pour déranger l'homme dans son travail, est transformé par Dieu en cheval ${ }^{51}$ : par là, la force diabolique est changée en bien. Un sens caché similaire anime tous les objets et les phénomènes avec lesquels l'homme est confronté au quotidien. Et c'est pourquoi la vie de tous les jours est présentée comme importante non pas en soi, mais en tant que rapport constant des mondes indissolubles, le sacré et le profane, ainsi que comme la réalisation de cette indissolubilité. 
Du point de vue du décodage des sens cachés, le conte "Tout n'entre pas dans la tête » présente le plus grand intérêt. Un certain jeune homme, qui voulait savoir tout au monde, rencontra Dieu. " "Comment veux-tu tout savoir au monde alors que tu ne vois pas ce qu'il y a devant tes yeux ? Tout n'entre pas dans la tête", lui dit Dieu qui prit le garçon en apprentissage. $\aleph^{52} \mathrm{~S}^{2}$ étant élevé dansleciel, lediscipledeDieuvitdeschoses étonnantes : unmoujikqui étaitassisseulsuruneimmensehat ${ }^{53}$ etquicriait : «Oh, commec'est étroit !»; desmoutons épuiséspaissantdansunpré luxuriantetdesmoutonsgraspaissant sur une terre inculte ; un homme s'efforçant en vain d'attraper un oiseau ; et finalement, une charogne pourrissante juste aux portes du paradis. Il cracha de dégoût et alla chez Dieu, qui lui expliqua le sens tant ésotérique que moral des faits:

Voici comment se présente en bref le canevas du conte. Il y a néanmoins des détails sur lesquels je souhaiterais m'arrêter.

Toutd'abord, c'estl'hommequiattrapel'oiseau (l'âme) qui vole de nid en nid. Notre héros lui conseille de cacher un des nids avec sa main, mais après leur nombre est multiplié par deux. Dieu juge l'acte du jeune garçon ainsi : "Tu voulais l'aider et tu as fais pire. $»^{55}$ L'hommedoitfaireseullechoixentrelebienetlemal (lesnids) etuneaideextérieureempêche de faire un tel choix. Cela ajoute des couleurs supplémentaires à l'image du paysan biélorusse : en raison de tous ses asservissements de l'extérieur, l'homme est compris comme une créature intérieurement libre et autosuffisante.

Le deuxième détail remarquable du conte est le moment où Dieu donne son appréciation sur le crachat du héros. Il semblerait qu'il devait imposer au jeune homme le dégoût pour la charogne (pour les cadavres de ses parents sorciers). Toutefois, ce geste est jugé comme un péché. Ici, nous avons affaire à la collision de valeurs de niveaux différents : la valeur des parents dans la culture biélorusse traditionnelle est d'autant plus importante qu'elle vainc l'aspiration à une pureté morale. Outre cela, le souhait de ne pas être sali par le péché a, pour le Biélorusse, sans doute moins d'importance que la charité, la compassion. En témoigne l'épisode où le garçon descend en enfer et, au lieu de ressentir du dégoût pour les âmes pécheresses qui se tordent dans le chaudron de goudron, il les prend en pitié :

Soulignonsquelechangementdusortdespécheursdépenddelaprièredel'homme, enaccord, nonpasformelmaissubstantiel, aveclesidéauxchrétiensdupardonetdusalut. Rappelons également que l'homme, tout comme Dieu, est capable de lancer le mécanisme apocalyptique de transformation du mal en bien. Toutefois, nous insistons encore, le mécanisme à l'aide duquel le héros du conte transfigure la situation n'est pas une action, mais une bonne pensée: la pitié et la compassion. Si nous approfondissons ce raisonnement, nous en arrivons à expliquer cette qualité que les chercheurs appellent "mollesse sociale ", "passivité ", «fatalisme », « indifférence» et qu'ils rapportent fréquemment au «caractère national» biélorusse. Le paysan biélorusse n'est pas indifférent : il est persuadé que les changements pour un mieux sont possibles, avant tout grâce à l'autoéducation et au perfectionnement moral. D'où, le Biélorusse n'est pas enclin aux transformations globales de la réalité: il comprend que seule une révolution anthropologique intérieure aidera à améliorer le monde. La charité rééduque le pécheur et l'oblige à honorer Dieu : ainsi, une marraine, qui, parce qu'elle a abandonné un petit orphelin à son sort, souffre mortellement dans le chaudron de l'enfer, est libérée sur sa parole $\left(« G u s l i »^{57}\right)$. C'est sans doute pourquoi, dans les contes biélorusses, la seule exception qu'est l'image du pan n'est, en fait, pas la représentation de gens tout à fait corrompus ${ }^{58}$. Etsilemalnevientpasdesgens, maisdelaloiinébranlable 
quiprédéterminelechangementconstantdubienenmaletviceversa,

alorslamanière detraitersaproprevieetcelledesautresdevient prudente, détournée.

Iln'estpaspossibledevaincreouvertementl'injusticedelavie, onnepeutquel'acceptercommedû. On peut améliorer la vie par des mesures locales, dans des lieux particuliers : sur sa parcelle de vie, dans son cercle, dans sa famille. Dans le cas extrême, on peut « rouler " l'injustice et ainsi la vaincre, non pas de manière rationnelle, mais en pénétrant au fond de l'être. Or, ce n'est possible qu'en refusant l'ingérence dans le cours des choses et dans les réalisations des tâches données par Dieu, non pas l'essence transcendante, ni l'icône à l'église, mais le Dieu paysan qui apparaît à l'homme « comme un frère ».

\section{NOTES}

1. Černjavskaja J. V., Belorus: štrihi kavto-portretu (Etničeskij samoobraz belorusa vskazkah) (Le Biélorusse: traits pour un autoportrait [Autoimage ethnique de Biélorusse dans les contes]), Éd. Četyre četverti, Minsk, 2006, 244 p.

2. Il faut ajouter que le processus d'identification religieuse sur le territoire de la Biélorussie a été compliqué par une certaine différence de conceptions des populations des régions occidentales (tradition-nellement plus catholiques) et centrales, mais aussi orientales (principalement orthodoxes). Dans le présent article, nous allons étudier plus spécialement les contes des régions orientales et centrales de la Biélorussie.

3. Weber M., «Obektiv-noš̌ poznanija voblasti socjal'nyh nauk i socjal'noj politiki » (L'objectivité de la connaissance dans le domaine des sciences sociales et de la politique sociale), in:Kul'turologija. XX vek (Culturologie. Le XXe siècle), Univer-sitetskaja kniga, Moscou, 1995, pp. 557-603.

4. Mead M., Metraux Ph., The study of Culture at a Distance (L'étude de la culture à distance), University of Chicago Press, Chicago, 1953, $480 \mathrm{p}$.

5. Idem ; Gorer G., Rickman J., The people of Great Russia : a Psycho-logical Study (Le peuple de Grande Russie : une étude psychologique), The Gresset Press, London, 1949, 235 p.; Dubois C., The People of Alor: a Socio-Psychological Study of an East Indian Island (Le peuple d'Alor: une étude sociopsychologique d'une île indienne orientale), University Minnesota Press, Minneapolis, 1944, 654 p. ; Benedict R., Hrizantema I meč : modeli japonskoj kul'tury (Le chrysanthème et le saber : les modèles de la culture japonaise), ROSSPEN, Moscou, 2004, 256 p. ; Mead M., Kul'tura I mir detstva. Izbrannye proizvedenija (La culture et le monde de l'enfance. CEuvres choisies), Nauka, Moscou, $1988,429 \mathrm{p}$.

6. Bogdanov K., Povsednevnoš̌ i mifologija. Issledovanie po semiotike for'klornoj dejstvuteL’nosti (Le quotidien et la mythologie. Recherches sur la sémiotique de la réalité folklorique), Isskustvo, Saint-Pétersbourg, 2001，438 p.; Nekljudov S. J., «Istoričeskij narrativ: meždu "reaL’noj dejstvitel'nosŤju" i foz'klorno-mifologičeskoj shemoj » («La narration historique : entre "réalité réelle" et schéma mytho-folklorique »), in : Mifologija i povsednevnosŤ (Mythologie et quotidien), RGGU, Saint-Pétersbourg, 1998, pp. 288-292 ; Nekludov S. J., Struktura i funkcija mifa (Structure et fonction du mythe), in : Mify v sovremennoj Rossii (Les mythes dans la Russie moderne), AIRO-HH, Moscou, 2000, pp. 17-38 ; Meletinskij E. M., Poetika mifa, Vostočnaja literatura RAN, Moscou, 1995, 
408 p. ; Propp V. J., Morfologija skazki (La morphologie du conte merveilleux), Nauka, Moscou, 1969,128 p. ; etc.

7. Bourdieu P., Načala (Le début), Socio-Logos, Moscou, 1994, pp. 193-194.

8. Du reste, nous sommes loin de considérer le paganisme comme une « religion véritablement biélorusse ", comme le pensent de nombreux chercheurs biélorusses contemporains, qui l'opposent au christianisme. À notre avis, de telles opinions ne se basent pas tant sur des objectifs scientifiques qu'idéologiques : ils opposent à l'orthodoxie "russe » le paganisme "biélorusse ", réclamé en qualité de base métaphysique pour se distancier de la Russie contemporaine. De cette manière, nous avons affaire ici à un phénomène de «téléologie rétrospective " (termes de Louis Althusser).

9. Dans la mythologie russe, le gumennik est un esprit démoniaque de la grange (gymno), lieu où l'on séchait le blé. Le gumennik peut y mettre le feu s'il est mécontent. (NdT)

10. L'ovinnik est un esprit féroce qui se cache dans le séchoir à blé. Selon les régions, il est représenté sous les traits d'un chat noir aux yeux noirs, d'un mouton ou d'un mort. Il est difficile d'amadouer l'ovinnik, dont le rôle principal est de surveiller la grange. La croyance aux méfaits de l'ovinnik vient du fait que le séchoir à blé (ovin) fonctionne avec un feu ouvert, lequel a plus d'une fois fait des ravages. (NdT)

11. Le hlevnik est l'esprit de l'étable (hlev). (NdT)

12. Sous ses apparences de belle jeune fille, la rusalka est une ondine, proche de la sirène, vivant dans les rivières. $(\mathrm{NdT})$

13. Esprit malin des eaux, le vodjanik est aussi appelé vodjanoj. Ressemblant à un vieillard, il pousse les gens dans l'eau et les noie. (NdT)

14. Seržputojskij A. K., Prymh i zabobony belarusaj-palešukoj (Croyances et superstitions du peuple biélorusse polésien), Universiteckae, Minsk, 1998, p. 236.

15. Idem.

16. Legendy i padannyi (Légendes religieuses et légendes locales), Grynblat M. \& Gruski A. (dir.), Navuka i Tehnika, Minsk, 1983, p. 40.

17. Seržputojski A. K., Kazki i anavjadanni belarucaj Sluckaga poveta (Contes et récits du district de Sluck), Universiteckae, Minsk, 2000, p. 105.

18. Idem.

19. Seržputojski A. K., Prymh... Op. cit., p. 125.

20. En Biélorussie, par le mot russe « hozjajstvo » ("gaspadarka » en biélorusse), on entend la propriété paysanne (la maison, la parcelle de terre, le potager, le bétail domestique, etc.).

21. Seržputojski A. K., Kazki..., Op. cit., p. 74.

22. Idem.

23. Ibid., p. 76.

24. Seržputojski A. K., Prymh..., Op. cit., p. 237.

25. Ibid., p. 285.

26. Seržputojski A. K., Kazki..., Op. cit., p. 213.

27. Jurij Lotman a appelé les serviteurs du culte des "médiateurs entre le monde et la vérité ». Voir : Lotman J. M., “ "Izgoj” i "izgojničestvo” kak socjal'no-psikhologičeskaja pozicija v russkoj kul'ture preimuščestvenno dopetrovskogo perioda " (Le "proscrit" et la "proscription" comme une position socio-psychologique dans la culture russe principalement pendant la période d'avant Pierre $\mathrm{I}^{\mathrm{er}}$ ), in: Istorija i tipologija russkoj kul'tury (Histoire et typologie de la culture russe),Iskusstvo, Saint-Pétersbourg, 2002, p. 225.

28. Seržputojski A. K., Prymh..., Op. cit., p. 72.

29. Idem.

30. Ce mot vient de la mythologie slave. Aux temps du paganisme, le monde se divisait en « jav' » et en "nav' ", en un monde réel et un monde métaphysique, magique. Quand les Slaves de l'Est se sont convertis au christianisme, le «jav'» est tout simplement devenu le monde d'en bas, celui 
dans lequel les hommes vivent. Par contre, le «nav' " est devenu le monde des enchantements, de la sorcellerie, le monde des anciens dieux païens, des rusalki (voir note 11) et des sorcières. Dans le présent contexte, l'auteur veut dire que le prêtre combine ses propres fonctions avec celle de chamane. (NdT)

31. Ulaščyk M., Vybranae (๕uuvres choisies), Belaruski knigazbor, Minsk, 2001, p. 31.

32. Seržputojski A. K., Kazki..., Op. cit., p. 238.

33. Ibid., pp. 65-66.

34. Ibid., p.177.

35. Ibid., p.42.

36. Ibid., p.260.

37. Ibid., p.201.

38. Buber M., Dvaobrazavery(Deuxformesdefoi), trad. del'allemandparGurevič P. S., Levit S. J. etLezov S. V., IzdateL’stvo«Respublika », Moscou, 1995.

39. Seržputojski A. K., Kazki..., Op. cit., p.108.

40. Ibid., p.185.

41. Ibid., p.56.

42. Ibid., p.57.

43. Ibid., pp. 175-177.

44. Ibid., pp. 112-116.

45. Ils'agitd'unbortnik, c'est-à-dired'un apiculteur des bois. Il ne prélève que le miel d'abeilles des bois.

46. SeržputojskiA. K., Kazki..., Ibid.p.239.

47. Ibid. p.240.

48. Ibid., p.243.

49. Lepragmatismeestsouventconsidéré commeunedesqualitésmentalesdebaseduBiélorusse : ilapparaît comme un topos dans son genre dans les recherches nationales et internationales.

50. Seržputojski A. K., Kazki..., Op. cit., pp. 76-77.

51. Legendy i padannyi, Op. cit., p.443.

52. Seržputojski A. K., Kazki..., Op. cit., p.221.

53. Habitationpaysanned'Ukraine. (NdT)

54. Seržputojski A. K., Kazki..., Op. cit., p.223.

55. Idem.

56. Idem.

57. Instrument de musique à cordes pincées. $(\mathrm{NdT})$

58. Même le pope se change parfois pour un mieux. Cela se produit le plus souvent quand il est pauvre et donc proche du paysan, par sa situation matérielle. Par là, la pauvreté est comprise comme une condition et un engagement de probité.

INDEX

Index géographique : Biélorussie

Mots-clés : paysannerie, religion, religiosité 


\section{AUTEURS}

\section{JULJA ČERNJAVSKAJA}

Professeur extraordinaire de l'Université biélorusse d'État de Culture et d'Art de Minsk 